研究

\title{
Contamination of Auditory Evoked Potential of Goldfish Carassius auratus with Microphonic Potential
}

\author{
Tomohiro SUGA (Graduate School of Fisheries Sciences, Hokkaido University) \\ Tomonari AKAMATSU (National Research Institute of Fisheries Engineering) \\ Tomonori HIRAISHI, Katsutaro YAMAMOTO \\ (Graduate School of Fisheries Sciences, Hokkaido University) \\ tomohiro@fish.hokudai.ac.jp \\ (Received 8 August 2005)
}

\begin{abstract}
:
In this experiment, we used sound stimuli of different durations to demonstrate that the auditory evoked potential (AEP) of fishes was contaminated with microphonic potential. We demonstrated that the AEP contained a component that had double the frequency as that of the sound stimulus. The durations of the AEPs were in proportion with the durations of the sound stimuli. "Auditory brainstem response" has been considered to be the dominant component of the AEP of fishes. However, this study suggests that some of the AEP components of fishes are derived from peripheral organs such as the saccule, lagena and utricle in the inner ear.
\end{abstract}

Classification: Fishery and Bioacoustics

Keyword: Auditory brainstem response, Fish hearing, Microphonic potential, Auditory evoked potential

\section{Introduction}

Recently, auditory evoked potential has been used to measure the hearing abilities of fishes ${ }^{1-3}$. The benefits of this methodology are as follows: no surgery is required and an auditory response can be easily obtained by placing an electrode on the head surface of the fishes. The low invasiveness and simplicity of this procedure of measurement enables repeated tests on the same test subject and the measurement of the hearing abilities of small test subjects such as juvenile fishes.

The origin of the auditory evoked potential has been studied well in human beings. One of the auditory evoked responses in humans, namely, the auditory brainstem response, originates from the midbrain or cerebrum, and this response is unrelated to the duration of the sound stimulus ${ }^{4}$, $)$. Another auditory evoked potential in humans, namely, the frequency following response, occurs in the cochlea or midbrain in a manner similar to the cochlea microphonic potential; these responses produce waveforms of identical shape for sound stimuli and are related to the duration of the stimulus ${ }^{6}$. 
In goldfish, it is known that the microphonic potential of the sacculus of the inner ear has doubled the frequency of the sound stimulus and is influenced by the duration of the stimulus. It is also known that in goldfish, the frequency following response of the sacculus nerve is equal to or double the frequency of the sound stimulus and is influenced by the duration of the stimulus $^{7)}$.

However, the origin of the auditory evoked potential (AEP) in goldfish is poorly understood thus far. To date, there have been no reports that focus on the origin of the AEP in goldfish. In this experiment, we examined the waveform of the auditory evoked potential by exposing goldfish to sound stimuli of different durations and comparing the observed waveforms to those of the microphonic potential.

\section{Materials and Method}

Figure 1 shows a diagram of the experimental system. Five goldfish, whose total body lengths ranged from $44.1 \mathrm{~mm}$ to $74.2 \mathrm{~mm}$, were used in this experiment. Sound stimuli were generated using a software (Cool Edit 2000) that can edit and produce sound waves and a connected attenuator (Kenwood RA$920 \mathrm{~A})$ that reduces the sound pressure level to a $10-\mathrm{dB}$ step or 5-dB step. An audio amplifier (Pioneer AC3) was used to amplify the sound signal. A tone burst

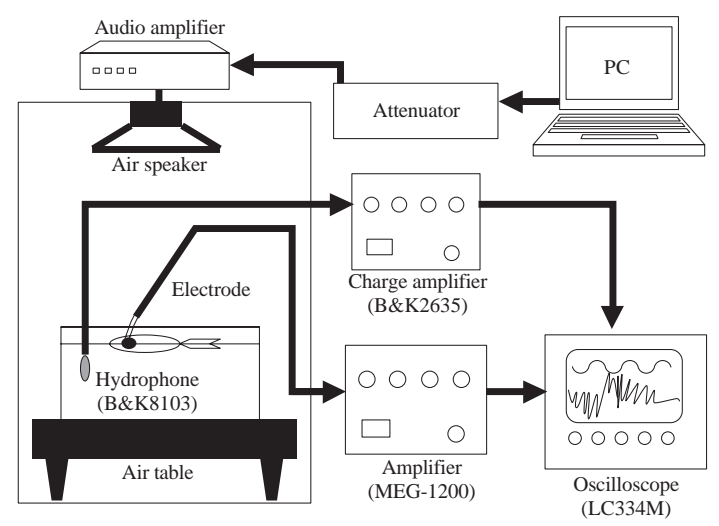

Fig. 1 diagram of experiment system. stimulus of $1 \mathrm{kHz}$ and a sound pressure level of 120 $\mathrm{dB}(\mathrm{dB}$ re $1 \mu \mathrm{Pa})$ were used for the test signal. The interval between the sound stimuli was set at $500 \mathrm{~ms}$. The phase of each burst alternated by 180 degrees to eliminate phase lock artefact. The durations of the sound stimuli used were $1 \mathrm{~ms}, 5 \mathrm{~ms}, 10 \mathrm{~ms}$ and $20 \mathrm{~ms}$.

The sound stimuli were measured using a B\&K8103 hydrophone (Bruel \& Kjaer Co). The test sounds were recorded by the hydrophone placed near the subject, and the electric signals from the hydrophone were amplified using the B\&K 2635 charge amplifier (Bruel \& Kjaer Co) and recorded in an oscilloscope (LC334M; LeCroy Co).

A small test aquarium $(34 \times 20 \times 24.5 \mathrm{~cm}$ in dimension; length $\times$ width $\times$ height) was placed on an air table to prevent any contamination by vibrations from the floor of the laboratory. The test aquarium was placed in a soundproof room to eliminate any noise from the environment. The test fish were wrapped in neoprene rubber and fastened using a plastic clip with only the head of the fish exposed. The fish were then held with a hand-shaped clamp that was supported by a prop. Teflon-coated tungsten wires $(0.1 \mathrm{~mm})$ were used for recording the auditory responses. The recording electrode was placed on the midbrain region of the head surface and a reference electrode was placed 5 $\mathrm{mm}$ in front of the recording electrode ${ }^{2)}$. A ground line was placed in the water of an aquarium. The auditory evoked potential produced in response to the sound stimuli was amplified by an amplifier (MEG-1200; Nihon Kohden). On an average, 300 waveformes were recorded by the oscilloscope.

\section{Results}

We could record the auditory evoked potential produced in response to all the sound stimuli, i.e. $1 \mathrm{~ms}, 5$ $\mathrm{ms}, 10 \mathrm{~ms}$ and $20 \mathrm{~ms}$. Figure 2 shows the AEP responses to each sound stimulus. The duration of the AEP approximately corresponded to the duration of 

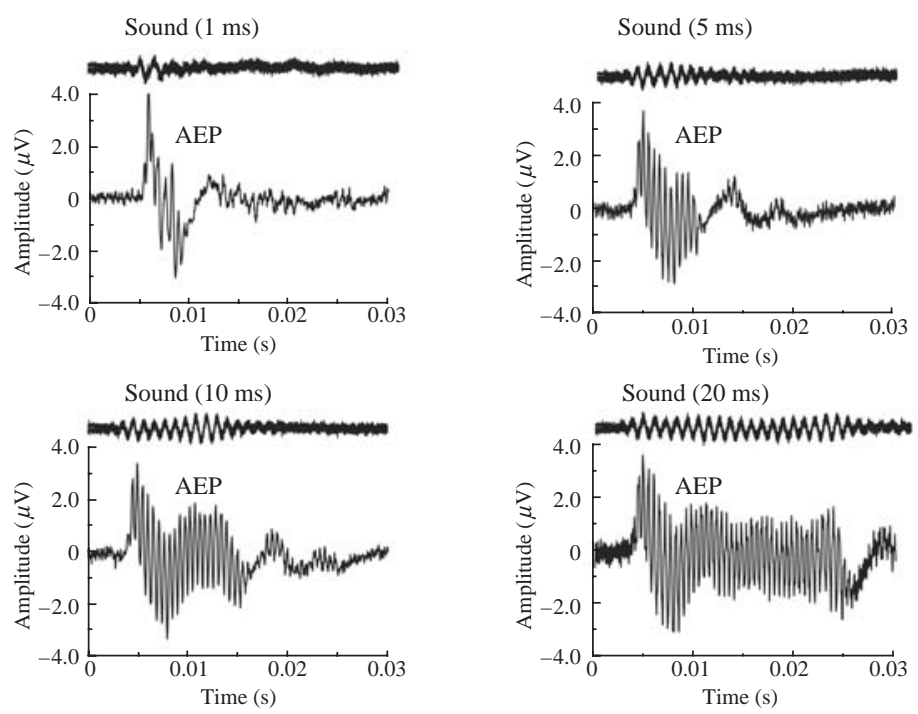

Fig. 2 AEP responses to each duration test sound stimuli.

each sound stimulus, except for the AEP to the 1-ms clicking sound stimulus. The duration of the AEP to a clicking sound was approximately $5 \mathrm{~ms}$. Figure 3 shows a Fourier transform of the AEP response to sound of $1-\mathrm{kHz}$ frequency and $20-\mathrm{ms}$ duration. The dominant component of all the AEP responses to sound stimuli of more than 5-ms duration was at approximately $2 \mathrm{kHz}$, which was double the frequency of the projected sound, i.e. $1 \mathrm{kHz}$. The AEP responses to the 1-ms clicking sound stimuli did not have a predominant $2-\mathrm{kHz}$ component but had a broadband frequency component ranging from $1 \mathrm{kHz}$ to $3 \mathrm{kHz}$. The peak-to-peak amplitudes of all the AEP waveforms were less than approximately $5 \mu \mathrm{V}$. The AEP waveforms produced in response to all the test sound stimuli were biased positive and then negative at the onset of the response. All the AEP waveforms produced in response to the 1-ms clicking sound stimulus differed from the AEP responses to sound stimuli with a duration of more than $5 \mathrm{~ms}$. AEP waveforms with a similar bias were detected in a previous study ${ }^{8)}$. The tested dead goldfish did not respond to the sound stimuli, and no AEP waveforms were recorded for them.

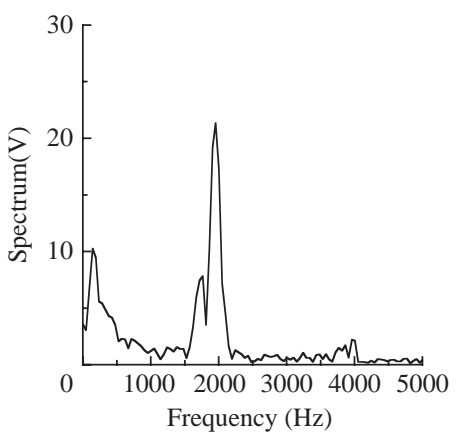

Fig. 3 Fourier transform ofAEP response to $1 \mathrm{kHz} 20 \mathrm{~ms}$ sound.

\section{Discussion}

In this experiment, we compared the AEP frequency, which included the doubled frequency component, to the sound stimulus frequency; these responses were equal to the durations of the sound stimuli, except in the case of the AEP response to the sound of $1 \mathrm{~ms}$ duration. And the AEP response to 1-ms clicking sound stimulus contains a broadband frequency component. The hair cells of the inner ear could be activated by these broadband frequencies. This might be one of the reasons behind our not observing a doubled frequency component in the AEP to 1-ms clicking sound stimulus. 
It is reported that microphonic potential, which has a frequency double that of the sound stimulus and has a sinusoidal waveform relating to the sound duration, can be recorded from the inner ear saccule when the recording electrode is inserted into this saccule in goldfish $^{7}$. In addition, responses that has a frequency double as that of the sound stimulus were measured in the lagena and utricle of the sleeper goby ${ }^{9}{ }^{10}$. The AEP recorded in this experiment was similar to the responses described above. We consider that the AEP recorded by placing electrodes on the midbrain region of the head surface, as in this experiment, is derived from the peripheral organs such as saccule, lagena and utricle in the inner ear or midbrain of the fish.

However, it is not yet apparent whether the origin of the AEP in this experiment is only the saccule or also includes higher process mechanisms involving the diencephalons, midbrain or cerebrum. There are few studies regarding the mechanisms of these origins of AEP in fishes, except for galeomorph sharks. It is known that the saccule and the eighth nerve are related to AEP to sound stimuli in galeomorph sharks ${ }^{11}$. Further, the origin of AEP appears to involve the medulla, midbrain and eighth nerve in this shark because it is reported that when the midbrain or eighth nerve is cut, the AEP changes slightly ${ }^{11)}$. Thus, galeomorph sharks have higher process mechanisms to code sound stimuli.

\section{Acknowledgments}

We thank Laurel Knapp for helping our experiment. We thank M. Ota, Y. Takao, T. Okumura, H. Saitou and A. Matsuda, researchers at the National Research Institute of Fisheries Engineering and the Program for Promotion of Basic Research Activities for Innovative Biosciences of Japan, for providing facilities and funding.

\section{References}

1) T. Akamatsu, A. Nanami, H. Y. Yan, "Spotlined sardine Sardinops melanostictus listens to $1-\mathrm{kHz}$ sound by using its gas bladder," Fish. Sci., 69, 348-354 (2003).

2) T. N. Kenyon, F. Ladich, H. Y. Yan, "A comparative study of hearing ability in fishes: the auditory brainstem response approach," J. Comp. Physiol. A, 182, 307-318 (1998).

3) L. E. Wysocki, F. Ladich, "The representation of conspecific sounds in the auditory brainstem of teleost fishes," J. Exp. Biol., 206, 2229-2240 (2003).

4) K. Kaga, "Hito to Doubutu no hakei to kigen", in Handbook of ABR, K. Kaga Eds. (Kanehara Shuppan, Tokyo, 1998), p. 9-13.

5) K. Ito, "Shigekion ni yoru ABR hakei", in Handbook of ABR, K.Kaga Eds. (Kanehara Shuppan, Tokyo, 1998), p. 64-66.

6) R. Batra, S. Kuwada, V. L. Maher, "The frequency following response to continuous tones in humans," Hear. Res., 21, 167-177 (1986).

7) T. Furukawa, "Chokaku", in Gyorui Seiri, Kawamoto Eds. (Kouseishya Kouseikaku, Tokyo, 1997), p. 491-512.

8) L. E. Wysocki, F. Ladich, "Can fishes resolve temporal characteristics of sounds? New insights using auditory brainstem responses," Hear. Res., 169, 36-46 (2002).

9) Z. Lu, Z. Xu, W. J. Buchser, "Acoustic response properties of lagena nerve fibers in the sleeper goby, Dormitator latifrons," J. Comp. Physiol. A., 189, 889-905 (2003).

10) Z. Lu, Z. Xu, W. J. Buchser, "Coding of acoustic particle motion by utricular fibers in the sleeper goby, Dormitator latifrons," J. Comp. Physiol., 190, 923-938 (2004).

11) J. T. Corwin, "Audition in Elasmobranchs", in Hearing and sound communication in fish, W. N. Tavolga, A. N. Popper, R. R. Fay Eds. (Springer-Verlag, New York, 1981), p. 81-105. 